\title{
Testing GPS generated 1PPS against a rubidium standard
}

\author{
Vukan Ogrizović ${ }^{1}$, Jadranka Marendić ${ }^{2}$, Snežana Renovica ${ }^{2}$, Siniša Delčev ${ }^{1}$, Jelena Gučević ${ }^{1}$ \\ ${ }^{1}$ University of Belgrade, Faculty of Civil Engineering, Bulevar kralja Aleksandra 73, 11000 Belgrade, Serbia \\ ${ }^{2}$ Directorate of Measures and Precious Metals, Mike Alasa 14, 11000 Belgrade, Serbia
}

ABSTRACT

GPS receivers are often used in time-keeping applications, due to their availability, low price and high accuracy. We tested the capability of the GPS receiver to deliver a time-keeping accuracy needed for the time-critical applications, such as astrometry measurements, when a microsecond or better level of accuracy is needed in real-time. We tested a geodetic class GPS receiver against a rubidium standard, over a 24 hours period. In the overall view, the accuracy corresponds to the nominal values. However, we experienced outliers with a certain regularity that we could not explain with cycle-slips or the experimental set-up.

Keywords: GPS, 1PPS, time-keeping

Citation: Vukan Ogrizović, Jadranka Marendić, Snežana Renovica, Siniša Delčev, Jelena Gučević, "Testing GPS generated PPS against a rubidium standard", Acta IMEKO, vol. 2, no. 1, article 5, August 2013, identifier: IMEKO-ACTA-02(2013)-01-05

Editors: Paolo Carbone, University of Perugia, Italy; Ján Šaliga, Technical University of Košice, Slovakia; Dušan Agrež, University of Ljubljana, Slovenia Received January $10^{\text {th }}, 2013$; In final form July $11^{\text {th }}, 2013$; Published August 2013

Copyright: @ 2013 IMEKO. This is an open-access article distributed under the terms of the Creative Commons Attribution 3.0 License, which permits unrestricted use, distribution, and reproduction in any medium, provided the original author and source are credited

Funding: This work was supported by the Ministry of Education and Science of the Republic of Serbia (Project of the technological development TR-36048)

Corresponding author: Vukan Ogrizović, e-mail: vukan@grf.bg.ac.rs

\section{INTRODUCTION}

Distributed industrial and technological systems request precise time synchronization between their sectors. Devices connected to each other by a communication link have to share the same time system in order to complete their tasks [1]. The tightest synchronization requirements lead to the need of highly accurate clock settings, that can be accomplished by means of GPS [2]. The components of the distributed system can easily use the readily available GPS time standard, wherever they are located.

Computers in a local or a wide-area network synchronize their times using Network Time Protocol (NTP). In such clientserver configuration time matching between the workstations should be much below a second. On a wide-area network (WAN), NTP provides time synchronization from 10-50 ms, nominally, while $1 \mathrm{~ms}$ absolute accuracy can be achieved if a GPS is used as the time source within a local area network (LAN) [3].

In order to test the accuracy of time synchronization using GPS, an experiment with five GPS receivers, connected to the same antenna, was performed [4]. All five clocks were synchronized with an accuracy better than 50 ns [4]. Here, direct measurement of 1 Pulse Per Second (PPS) was used, instead of the transmission of the clock corrections via NTP.
An example of a high-precision time-keeping is monitoring of bridge deviations due to a heavy load. The surveyors mount GPS receivers to the characteristic points on the bridge. Receivers collect data for a long period under different loads. The relative accuracy of baseline vectors between the receivers depends on a lot of factors that decrease the accuracy of the vectors. Since the field crew do not have any impact on ambient conditions, the accuracy can be increased only by adapting the measuring method. Time synchronization is a tool for determining of pseudo-ranges and phase differences.

Accurate time is necessary for the astrometry applications, like the determination of the Earth's orientation [5]. Also, due to the Earth's rotation, relative positions of the observed radiosources or stars, depending on the measuring method, change constantly. An error of $100 \mathrm{~ms}$ in determining the epoch of the observed object corresponds to an error of tens of meters in determining the position at the Earth's surface.

In astrometry applications, an accuracy of the order of microseconds (or better) is requested. The GPS receivers manufactured for timing applications or geodetic class GPS receivers provide a 1 PPS signal with an accuracy of up to $10 \mathrm{~ns}$ for 5 day average values, according to the manufacturers' specification. This accuracy is often misunderstood as the accuracy in real-time, which is much worse due to the large noise in the GPS signal. Here we performed a 24-hours test of such GPS receiver, comparing its 1PPS against 1PPS of a 
rubidium standard. The test should show whether the GPS receiver can provide reliable, consistent and accurate time signals over a 24-hour period.

Many other applications need precise time-keeping: event reconstruction, synchrophasors, system time and frequency deviation, multi-rate billing, power quality incentives, time tagging recorded data, wide area measurement systems, travelling-wave fault detection, end-to-end relay testing [6], and even electronic betting systems [7].

In Section 2 we will present the background and theoretical concepts of our research, with the focus on time-keeping methods and the resolving of cycle slips that occur during GPS measurements. Also, we will give the necessary equations needed for calculating the parameters of the device under test. Section 3 gives the results of our experiment. We presented the experiment set-up and the measurements we performed. Section 4 comprises the relevant conclusions we have drawn from the results, with some remarks and suggestions for further research.

\section{BACKGROUND AND METHODS}

\subsection{Time-keeping and transmission of time}

Time marks are transferred to users by many means, including terrestrial or satellite systems [8]. The GPS receivers usually transfer their time via an NTP network, serving as a stratum 1. Depending on the configuration, the GPS receiver can work as a node in the network, delivering the time signals using the TCP/IP protocol, or directly via a serial or a USB port. Both methods assume the $24 / 7$ configuration, with regular corrections of the clock in computers being synchronized.

Several manufacturers of GPS equipment offer a special class of GPS receivers capable of delivering 1 PPS via a BNC output, disciplining a computer clock applied in the specific timing application. Those 1PPS outputs are often steered using the receiver's GPS data, and do not represent the 1PPS output of the internal clock [9]. The application is not ready-made, but rather a user writes a custom low-level routine, written in lowlevel $\mathrm{C}$ or, even, assembler [10].

The features of the operating system influence the latencies appearing during the transfer of time signals from the source of 1 PPS to the computer. For that reason, such time-keeping routine often runs under a Real-Time Operating System (RTOS). The RTOS treats software and hardware interrupts in a special way, minimizing the latencies. Synchronization by catching the PPS ticks with interrupts implies permanent synchronization with minimal latencies [11].

All methods used for time-keeping by the GPS receivers share the same issue. The configuration requires a minimum of 15 minutes after the start of the synchronization to adjust the computer clocks to required accuracy [9], [10], [12].

\subsection{Resolving cycle slips}

Cycle slips represent sudden jumps in GPS integrated phase measurements. When the cycle slip occurs, a new unknown appears in the mathematical model of an autonomous or relative solution. Increasing the number of unknowns degrades the internal reliability of the adjustment solution. Because of that, an extreme attention is paid to the cycle-slips resolving.

Here we applied the bias optimizing method [13]. If $b_{1}$ and $b_{2}$ are frequency biases for GPS frequencies L1 and L2, respectively, after the cycle slips in the amounts $\Delta n_{1}$ and $\Delta n_{2}$, new biases become $b_{1}^{\prime}$ and $b_{1}^{\prime}$, which can be written as:

$$
\left(\Delta n_{1}, \Delta n_{2}\right)=\left(b_{1}^{\prime}-b_{1}, b_{2}^{\prime}-b_{2}\right),
$$

For dual-frequency GPS receivers, a wide-lane linear phase combination $\Phi_{\delta}$ of carrier-phase data on frequencies $\Phi_{1}$ and $\Phi_{2}$ is created:

$$
\Phi_{\delta} \equiv\left(\Phi_{1}-\Phi_{2}\right),
$$

which, after the development given in [14], yields the bias of the wide-lane combination:

$b_{\delta}=\frac{1}{\lambda_{\delta}}\left(L_{\delta}-P_{\delta}\right)$,

where $\lambda_{\delta}$ is the wavelength, $L_{\delta}$ the carrier-phase data expressed in cycles, and $P_{\delta}$ the code pseudo-range. Subscript b stands for the wide-lane combination. The time-average value (3) is then calculated before and after the cycle slip. The difference between these two values should be close to an integer value:

$\Delta n \equiv\left(b_{\delta}^{\prime}-b_{\delta}\right)=\Delta n_{1}-\Delta n_{2}$.

\subsection{Determining the clock's stability}

We determined the short-term stability of the 1PPS output of the GPS receiver's clock. Commonly used measures of stability are different kinds of variances or their square roots deviations. We used the Allan variance [12]:

$\sigma_{y}^{2}(\tau)=\frac{1}{2 \tau^{2}}\left\langle\left(\Delta^{2} x\right)^{2}\right\rangle=\frac{1}{2}\left\langle(\Delta y)^{2}\right\rangle$,

or its square root $\sigma_{y}$ - Allan deviation (ADEV);

and Modified Allan variance [12],

Mod. $\sigma_{y}^{2}(\tau)=\frac{1}{2 \tau^{2}}\left\langle\left(\Delta^{2} \bar{x}\right)^{2}\right\rangle$,

or its square root - Modified Allan deviation (MDEV);

Time variance [12]

$\sigma_{x}^{2}(\tau)=\frac{\tau^{2}}{3} \operatorname{Mod} . \sigma_{y}^{2}(\tau)=\frac{1}{6}\left\langle\left(\Delta^{2} \bar{x}\right)^{2}\right\rangle$,

or its square root - Time deviation (TDEV);

and Overlapping Hadamard variance, following the notation given in [15]:

$H \sigma_{y}^{2}(\tau)=\frac{1}{6(N-3 m) \tau^{2}} \sum_{i=1}^{N-3 m}\left[x_{i+3 m}-3 x_{i+2 m}+3 x_{i+1 m}-x_{i}\right]^{2}$.

The overlapping Hadamard variance is used for characterization of rubidium oscillators, because the linear frequency drift, characteristic for rubidium oscillators, does not affect it [15]. Also, the power spectral density is calculated, with a population consisting of 86400 samples.

\section{RESULTS AND DISCUSSION}

\subsection{Device under calibration and measurement}

We tested a geodetic class, dual frequency GPS receiver Trimble NetRS (depicted as DCM in Fig. 1). The receiver collects the code and phase GPS data on 24/7 basis within the Serbian Continuously Operating Reference Stations (CORS) network. Since a computer running under Linux is incorporated in the NetRS, it is attached directly to the Serbian Academic Network and, thus, available throughout the Internet.

The GPS antenna is mounted to the highest point of the roof of the building. No radio-sources or voltage cables exist in the vicinity of the antenna. We use a high-performance Trimble Zephyr Geodetic GPS antenna, connected to the receiver with 
a low-noise cable. Zephyr Geodetic antenna owns a groundplane, specially constructed for minimizing possible multipath effects. Further improvement could be made by constructing a counterpoise under the antenna.

Everyday use of NetRS is in surveying, serving as one of the nodes in the CORS network. However, it is equipped with a 1PPS output, which makes it convenient for time-keeping purposes.

The receiver delivers $8 \mu$ s wide pulses, with rise and fall times of 100 ns. The resolution is 40 ns, but factors like position uncertainty, antenna and cable delay uncertainty, may limit the accuracy of the 1 PPS signal up to $\pm 1 \mu$ s [16].

\subsection{Experimental set-up}

The experimental set-up is depicted in Figure 1, and the list of instruments used is given in Table 1.

One day prior to the start of the measurements, the rubidium frequency standard was calibrated against the Serbian time and frequency standard UTC(DMDM).

The $10 \mathrm{MHz}$ signal from the rubidium standard is divided down to 1PPS in the digital clock. The 1PPS from the digital clock is applied to the START input, the 1PPS from the DCM (NetRS GPS receiver) is applied to the STOP input of the time interval counter (TIC).

Input signal conditioning is as follows: DC coupling, $50 \Omega$, rising edge; trigger level set at $50 \%$ of the signal amplitude.

\subsection{Time interval measurement}

The frequency of the DCM is compared to the reference frequency by measuring the phase difference between the two 1 PPS signals and its change in time.

The phase difference is estimated by measuring the time interval between the zero crossings of the 1 PPS signals using the TIC. The relative frequency offset is calculated by fitting the least-square line to the data and taking the slope of the line.

The measurement time is $86400 \mathrm{~s}$.

The raw data diagram is given in the Figure 2.

The data acquisition and processing procedure was performed fully automatic. Time View software was used for data logging and processing.

The indoors ambient temperature was constant throughout the experiment, $20^{\circ} \mathrm{C}$.

During the 24 hours session, 16 outliers occurred depicted as sudden jumps in the phase-time diagram (Fig. 2). The outliers showed a certain regularity in the amount, which was

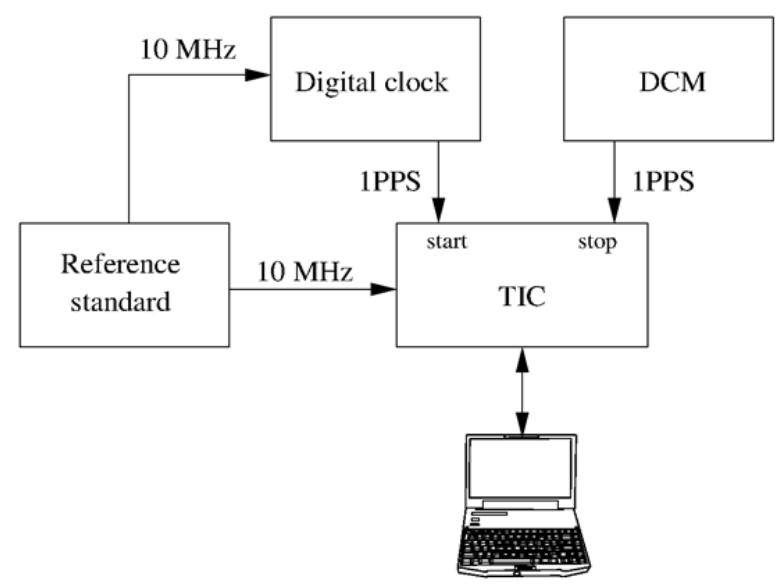

Figure 1. Experimental set-up.
Table 1: Equipment used in the experiment.

\begin{tabular}{lll}
\hline Name & Model & Manufacturer \\
\hline Rubidium frequency std. & $6689 / 021$ & Pendulum \\
Time Interval Counter & CNT-90 & Pendulum \\
NetRS & $6609 / 021$ & Trimble \\
Digital clock & CADM & Rohde-Schwartz \\
Oscilloscope & TDS 3032 & Tektronix \\
\hline
\end{tabular}

not expected. Their values were grouped around three points: approximately $-0.4 \mathrm{~ms},-0.6 \mathrm{~m}$, and $-1 \mathrm{~ms}$, in respect to the mean value.

After the removal of the outliers, and normalization (because the two 1 PPS signals were not synchronized) we obtain the plot in Fig. 3.

Fig. 4 presents the power spectral density.

Since the TIC CNT-90 has virtually zero dead-time between two successive sample measurements, stability features may be calculated from its measurement data. For this data processing we used Stable 32 software. In Fig. 5 various types of stability measures in the time domain (ADEV, MDEV, TDEV, and HDEV) are presented.

If the receiver is moving, the accuracy of its 1 PPS is decreasing [17]. Since the receiver used in the experiment is stationary, that source of error is neglected.

After the notification of the outliers, we processed the RINEX file with raw GPS data for cycle slips detection, in order to find the correlation with the outliers in the time-phase diagram. We followed the method described in [13], using the geometry-free and wide-lane linear combinations of raw dual frequency pseudorange and carrier phase data. We used the modules of GPS Toolkit [15], [18].

There were 56 cycle slips in total, during the 24 hours of data logging. We estimated the clock's stability independently by calculating Allan variances from RINEX data. The results are given in Fig. 6.

No correlation between the epochs of the cycle slips and outliers in 1PPS were found, which means that the satellite constellation did not affect the 1PPS performance.

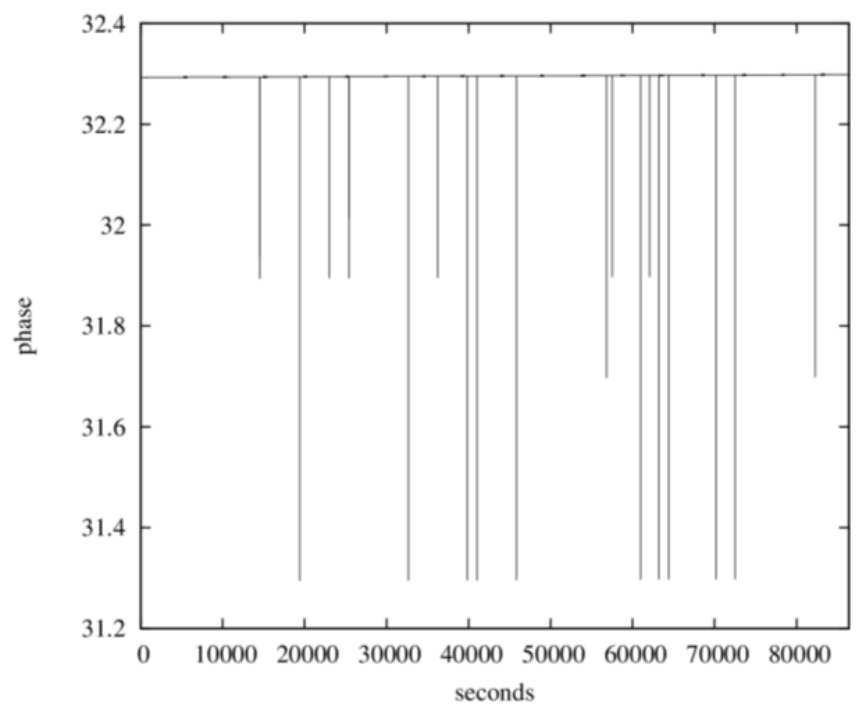

Figure 2: Time View graph. The two 1 PPS signals are not synchronized their difference is about $32 \mathrm{~ms}$. Apparent outliers. 
Considering the experiment set-up, ambient conditions (indoors and outdoors), examining the change of the satellite constellation, we did not find what caused the outliers in the 1PPS coming from the NetRS. They do not affect the everyday use of NetRS (surveying, positioning, and time-keeping in LAN), but could seriously degrade the accuracy of certain timecritical applications. Therefore, we are preparing the extension of the experiment, in order to investigate the problem.

\section{CONCLUSIONS AND REMARKS}

According to overall results of our 24 hours measuring session, it can be concluded that a geodetic class GPS receiver is capable of permanent delivering time marks within ms accuracy. Examples of such application are permanent monitoring of an industrial process, or time synchronization between distant computer nodes in a wide-area network.

Still, when higher accuracy is needed, in the order of microseconds or better, a special attention should be paid to the set-up of the equipment, keeping in mind what disturbances of the GPS signal could occur during the exploitation.

The outliers that occurred in our experiment could seriously degrade the accuracy of real-time applications. We did not find any relation between the obtained results and the experiment (set-up, ambient conditions, or satellite constellation). The grouping of the outliers in the mean of values and their irregularity considering the time epochs when they occurred, should be investigated further. We suggest another, longer experiment, which would last for seven days, in order to find the factor that causes outliers.

The mentioned outliers could degrade also the positional accuracy. The solution for high-precision surveying purposes is to discipline an external high stability oscillator, such as rubidium standard, with the GPS signal.

\section{ACKNOWLEDGEMENT}

The authors of the paper express their gratitude to the Ministry of Education and Science of the Republic of Serbia, who supported the work on this paper by participating in the project of technological development TR-36048.

\section{REFERENCES}

[1] J. Elson, D. Estrin, "Time synchronization for wireless Sensor networks", International Parallel and Distributed Processing Symposium (IPDPS), San Francisco (CA), USA, 2001, pp. 19651970.

[2] A. Carta, N. Locci, C. Muscas, F. Pinna, S. Sulis, "GPS and IEEE 1588 synchronization for the measurement of synchrophasors in electric power systems", Computer Standards \& Interfaces vol. 33, no. 2, 2011, pp. 176-18.

[3] H. Wohlwend, G. Crispieri, Y.S. Li, "Factory and equipment clock synchronization and time-stamping guidelines: version 2.0", International SEMATECH Manufacturing Initiative, Inc, 2008.

[4] K. Behrendt, K. Fodero, "The perfect time: an examination of time synchronization techniques", http://www.pespsrc.org/i/TP6226_PerfectTime_KB_20050922.pdf.

[5] D. McCarthy, "Using UTC to determine the Earth's rotation angle, in Decoupling Civil Timekeeping from Earth Rotation", Science and Technology Series, vol. 113, American Astronautical Society, ISBN 9780877035756, pp. 105-117.

[6] B. Dickerson, "Time in the power industry: how and why we use it", White paper, Arbiter Systems, http://www.arbiter.com/files/product-attachments/ TimeInThePowerIndustry.pdf.

[7] P. Hämälainen, M. Hämälainen, T.D. Hämälainen, R. Soininen, R. Rautee, "Design and implementation of real-time betting

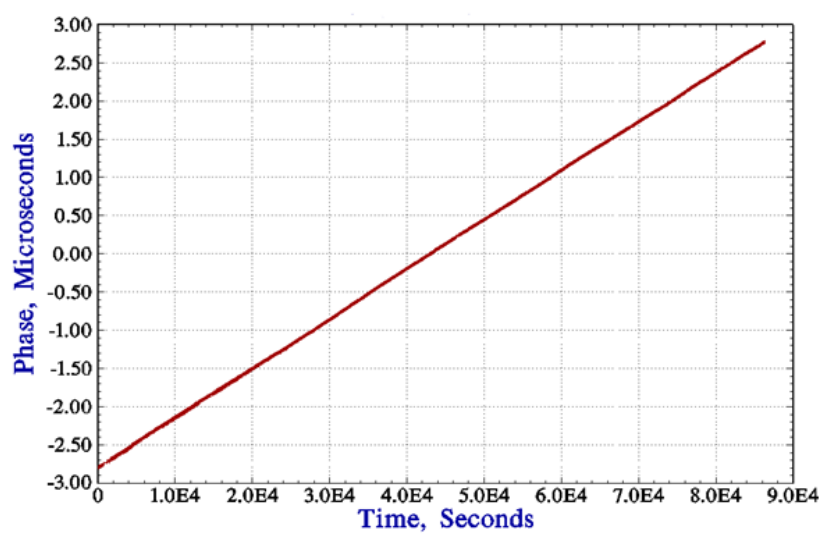

Figure 3. Phase-difference data after removal of outliers and normalization.

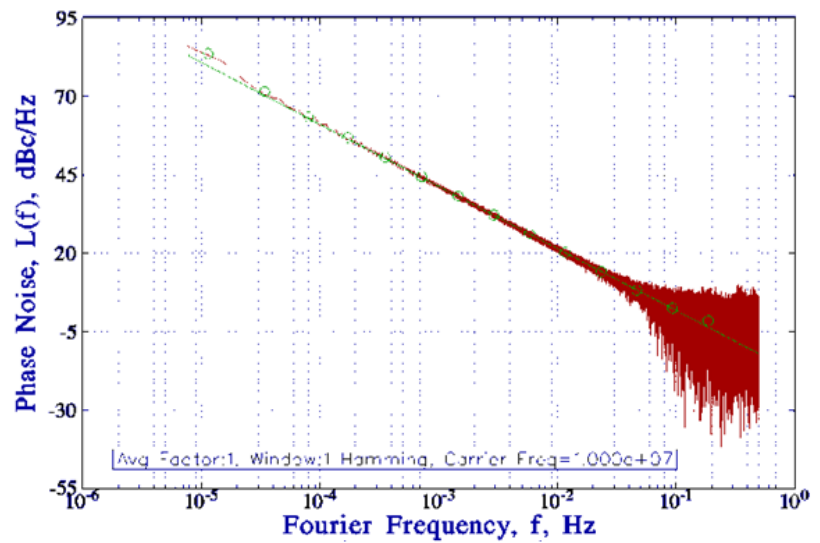

Figure 4. Power spectral density values.

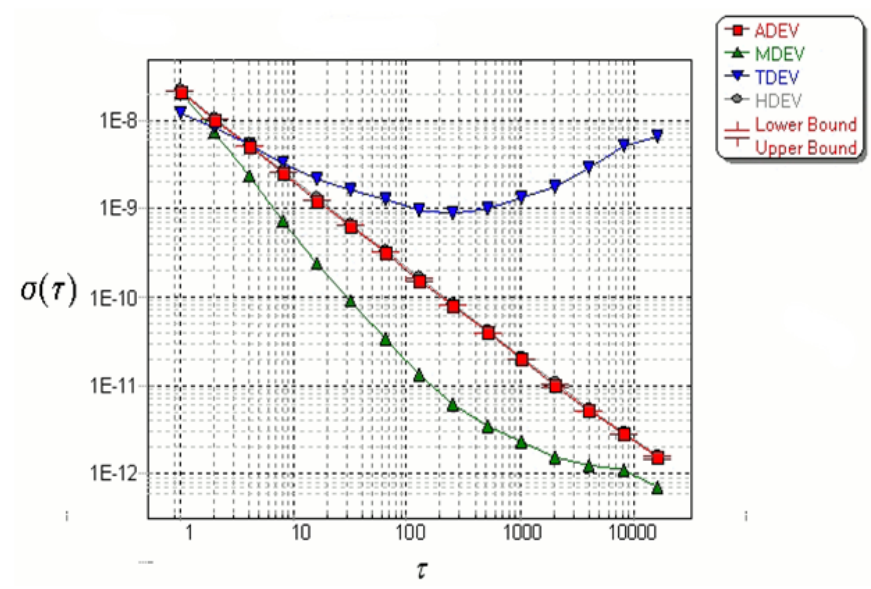

Figure 5. Stability measures of the 1 PPS output of the NetRS: Allan deviation, Modified Allan deviation, Time deviation, overlapping Hadamard deviation.

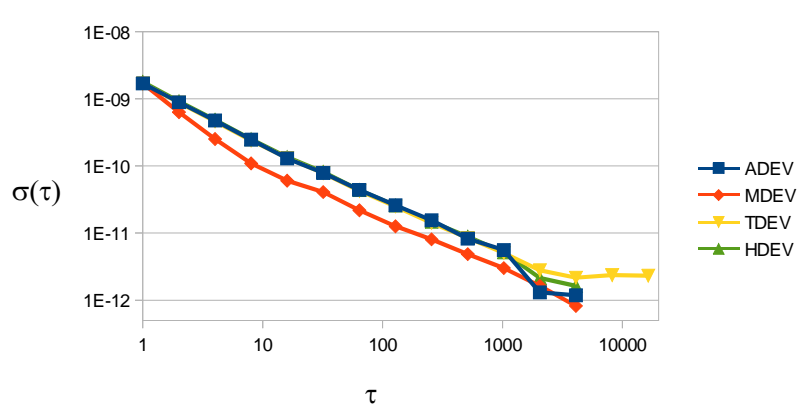

Figure 6. Allan variances from raw GPS data. 
system with offline terminals", Electronic Commerce Research and Applications vol. 5, no. 2, 2006, pp. 170-188.

[8] M. Lombardi, "NIST time and frequency services", NIST Special Publication 432, NIST, 2002.

[9] T. Schildknecht, G. Dudle, "Time and frequency transfer: high precision using GPS phase measurements", GPS World, Feb. 2000, pp.48-52.

[10] V. Ogrizović, "A construction of an advanced measuring system for astro-geodetic determinations", Publications of the Astronomical Observatory of Belgrade, vol. 86, 2009, pp. 145150.

[11] A. Heursch, "Using standard operating systems for time critical applications with special emphasis on LINUX", PhD Dissertation, Universität der Bundeswehr München, Fakultät für Informatik, 2006.

[12] D.W. Allan, N. Ashby, C.C. Hodge, "The science of timekeeping", Hewlett Packard Application Note 1289, 1997.

[13] G. Blewitt, "Carrier phase ambiguity resolution for the global positioning system applied to geodetic baselines up to $2000 \mathrm{~km}$ ",
Journal of Geophysical Research, vol. 94, no. 88, 1989, pp. $10,187-10,203$.

[14] G. Blewitt, "An automated editing algorithm for GPS data, Geophysical Research Letters”, vol. 17, no. 3, 1990, pp. 199-202.

[15] T. Craddock, R.J. Broderick, C.P. Petersen, A. Hu, "The GPS toolkit: open source clock tools", $40^{\text {th }}$ Annual Precise Timing and Time Interval (PTTI) Meeting, Reston (VA), USA, 2008, pp. 275274.

[16] "Trimble: NetRS GPS Receiver - User Guide", Trimble Navigation Limited, 2004.

[17] P. Hightower, "Motion effects on GPS receiver time accuracy", Instrumentation Technology Systems, Northridge (CA), USA, 2008.

[18] T. Gaussiran, D. Munton, B. Harris, B. Tolman, "An open source toolkit for GPS processing, total electron content effects", measurements and modelling, International Beacon Symposium, Trieste, Italy, 2004. 